KA - THEP - $4-1990$

Mannheimer Manuskripte 105/90

January 1990

\title{
Schwinger Terms from Geometric Quantization of Field Theories
}

\author{
H.EWEN ${ }^{1}$, P. SCHALLER ${ }^{1}$ and G. SCHWARZ ${ }^{2}$ \\ Mannheimer Manuskripte 105/90 \\ ${ }^{1}$ Institut für Theoretische Physik \\ Universität Karlsruhe \\ Kaiserstrasse 12, D.7500 Karlsruhe \\ W. - Germany \\ and \\ ${ }^{2}$ Lehrstuhl für Mathematik I \\ Universität Mannheim \\ Schloß, D - 6800 Mannheim \\ W. - Germany
}

\section{W....... ABSTRACT}

Geometric quantization is applied to infinite '(courtable) dimensional linear Kähler manifolds to obtain a closed expression for the anomalous commutator of arbitrary polynomial observables. Examples for the physical relevance of the result are given, including the polarization dependence of Schwinger terms in bilinear constraint algebras, the central terms of Virasoro and Kac-Moody algebras and the determination of the critical dimension of the bosonic string. 


\section{Introduction}

A significant feature of quantum theories on infinite dimensional phase spaces is the occurence of anomalies. Anomalous commutators between quantum operators, which appear in the algebra of first class constraints, are of special interest as they impose heavy restrictions on the formulation of a consistent quantum theory.

For finite dimensional systems the geometric quantization scheme [GuSt,Sni, Woo] is a well established tool, connecting the structure of the quantum theory with the geometry. of the classical phase space. It has also been applied to infinite dimensional systems in order to investigate anomalies [BoRa,SaSw], their connection to the vacuum structure of the quantum field theory [PIWa] and their polarization dependence [Nie].

The present paper is devoted to the calculation of anomalous commutators of polynomial observables on Kähler manifolds. All calculations are organized such that they hold for bosonic as well as for fermionic systems with finite or infinite (countable) dimenensions.

The central result, worked out in section 3 gives a closed expression for the anomalous commutator on a linear manifold. For bilinear observables this expression reduces to the trace of a matrix commutator and hence vanishes for finite dimensional systems. Applied to the constraint algebra of a field theory, as done in section 4 , it allows to calculate the Schwinger terms. The latter are shown to be cohomologically trivial if the (bilinear) constraints preserve the holomorphic structure.

The physical relevance of our results is demonstrated by application to the Kac-Moody and the Virasoro algebras in sections 5 and 6 , respectively. In section 7 we reconsider the BRS-scheme in the context of geometric quantization and calculate the square of the BRS-operator with our furmulas. Application to the bosonic string allows to determine its critical dimension. A graphical representation of our calculus reminding the technique of Feynman-graphs is presented in an appendix. 


\section{Geometric quantization}

Geometric quantization on Kähler manifolds is a well established feature in the literature [Sni, Woo]. Here we review only the main results, relevant for our study of anomalies. Let the phase space of a physical system be a symplectic manifold $(M, \omega)$, then prequantization means a representation of the Poisson algebra of classical observables - the smooth functions on $M$ - by operators on the Hilbert space of all smooth, square-integrable sections in a Hermitian line bundle $L$ over $M$. Such a prequantization is realized by a map

$$
F \mapsto \mathcal{O}_{F}=-i \hbar \nabla_{X_{\boldsymbol{F}}}+F
$$

where $X_{F}$ is the Hamiltonian vector field of $F$ and $\nabla$ a connection on $L$ with curvature $\operatorname{curv}(\nabla)=\omega / \hbar$. Let $M$ carry a complex structure $J$ and the symplectic form be determined (locally) by a Kähler potential as $\omega=d d^{+} \mathcal{K}\left(z, z^{+}\right)$, where $d$ and $d^{+}$denote the holomorphic and anti-holomorphic exterior derivative, respectively. Then we may choose

$$
\left.\nabla_{X}=X-X\right\rfloor\left(\frac{i\left(d^{+}-d\right)}{2 \hbar} \mathcal{K}\left(z, z^{+}\right)\right)
$$

To obtain an irreducible representation, however, one has to restrict the Hilbert space to the space $\mathcal{H}^{J}$ of holomorphic sections of $L$. More explicitly, denoting by $P^{J} \subset \Gamma(T M)$ the antiholomorphic polarization, spanned by the Hamiltonian vector fields of the coordinatefunctions $z_{k}$, then each quantum state $|\mathcal{S}\rangle$ is locally described by a function $\mathcal{S}\left(z, z^{+}\right)$on $M$, obeying $\nabla_{Y} \mathcal{S}=0 \forall Y \in P^{J}$. Furthermore the correct measure on $\mathcal{H}^{J}$ requires the introduction of (normalized) half forms $\nu_{0}$, i.e. the use of the metaplectic representation [Woo,GuSt] for the quantization. Then a quantum state $|\mathcal{S}\rangle$ is locally given by

$$
\mid \mathcal{S}>:=\mathcal{S}\left(z, z^{+}\right) \cdot \nu_{0}=\exp \left(\frac{i \mathcal{K}\left(z, z^{+}\right)}{2 \hbar}\right) \sigma(z) \cdot \nu_{0} \quad, \text { where } \quad \nu_{0}\left(X_{z_{1}}, \ldots X_{z_{k}}, \ldots\right)=1
$$

The operator $\mathcal{O}_{F}$ is in general not compatible with the polarization $P^{J}$, i.e. the action of $\mathcal{O}_{F}$ needs not to close in $\mathcal{H}^{J}$. For this reason prequantization has to be modified by the BKS-construction [Bla,Woo], relating quantum states described in different holomorphic structures. In the special case of a Kähler manifold this yields an explicit formula [Tuy1] for the quantum operator corresponding to an arbitrary (BKS-quantizable) observable $F$ :

$$
I F \mid \mathcal{S}>=\Pi^{\text {hol }}\left[\left(-i \hbar \nabla_{X_{\boldsymbol{P}}}+F\right) \mid \mathcal{S}>\right]
$$


where $\Pi^{\text {hol }}$ is the orthogonal projector onto the space of holomorphic sections $\mathcal{H}^{J}$. The projection is computed via convolution with a generalized Bergman kernel $\mathcal{B}\left(z, z^{+} ; y, y^{+}\right)$:

$$
\operatorname{IF}\left(\mathcal{S} \cdot \nu_{0}\right)\left(z, z^{+}\right)=\int \mathcal{B}\left(z, z^{+} ; y, y^{+}\right)\left(\mathcal{O}_{F}-\frac{i \hbar}{2} \operatorname{tr}^{J}\left(\mathcal{L}_{X_{F}}\right)\right) \mathcal{S}\left(y, y^{+}\right) d \mu_{\left(y, y^{+}\right)} \cdot \nu_{0}
$$

Here the Lie derivative $\mathcal{L}_{X_{F}}$ is considered as a linear operator on $\Gamma(T M)$ and $\operatorname{tr}^{J}$ is the trace taken over the holomorphic components. This contribution is due to the transformation of the half form under $\nabla_{X_{F}}$ and corresponds to the non-invariance of the volume form on the Hilbert space $\mathcal{H}^{J}$. The integration in (2.4) is done with the invariant measure

$$
d \mu_{\left(y, y^{+}\right)}=\left(\nu_{0}^{+} \nu_{0}\right) \sqrt{\left|\operatorname{det}\left(\kappa_{l n}\right)\right|} \prod_{k \in K} d y_{k} d y_{k}^{+} \quad \text { where } \quad \kappa_{k l}\left(y, y^{+}\right)=\partial_{y_{l}^{+}} \partial_{y_{k}} \mathcal{K}\left(y, y^{+}\right)
$$

and $\Pi d y_{k} d y_{k}^{+}$is the usual Lebesgue measure on $M$. The generalized Bergman kernel is given by the sum $\sum \varphi_{a}\left(z, z^{+}\right) \varphi_{a}^{+}\left(y, y^{+}\right)$, where $\left\{\varphi_{a}\right\}_{a \in A}$ is a complete set of orthonormal states in $\mathcal{H}^{J}$ [Tuy1]. Explicitly we obtain

$$
\mathcal{B}\left(z, z^{+} ; y, y^{+}\right)=\mathcal{N}_{0} \exp \left(\frac{i \mathcal{K}\left(z, z^{+}\right)}{2 \hbar}\right) \exp \left(\frac{i \Lambda\left(z, y^{+}\right)}{\hbar}\right) \exp \left(\frac{i \mathcal{K}\left(y, y^{+}\right)}{2 \hbar}\right)
$$

where $\mathcal{N}_{0}$ is a normalization and the function $\Lambda\left(z, y^{+}\right)$is determined by $\left\{\varphi_{a}\right\}_{a \in A}$. With certain assumptions we may integrate by parts in (2.3) and obtain:

Proposition [Tuy2]

If $M$ is a Kähler manifold, which is either compact without boundary or $\mathcal{S}\left(z, z^{+}\right)$decreases fast enough at $\partial M$, then the quantum operator corresponding to an observable $F$ is .

$$
\begin{aligned}
& I F \mid \mathcal{S}>=\Pi^{\text {hol }}\left[\left(F+\frac{i \hbar}{2} \Delta F\right) \mid \mathcal{S}>\right] \\
& \text { where } \quad \Delta F\left(z, z^{+}\right):=-\sum_{k, l \in K} \kappa_{k l}^{-1}\left(z, z^{+}\right) \vec{\partial}_{z_{l}^{+}} F\left(z, z^{+}\right) \overleftarrow{\partial}_{z_{k}}
\end{aligned}
$$

This local expression for the quantum operator is independent of the choice of coordinates.

As shown in [SaSw,Sch] it is possible to generalize this construction of [Tuy1,2]: To quantize (linear) field theories one starts with the (infinite dimensional) space of solutions of the classical field equations [Woo], considered as the phase space $M$. Moreover, for theories containing fermionic degrees of freedom, one can use the notion of graded manifolds [Kos,Ber] and proceed similarly to the bosonic case. Both generalizations do 
not change the form of the Bergman kernel (2.6), only the normalization constant $\mathcal{N}_{0}$ becomes formal. Furthermore in the graded case (2.4) is understood with the supertrace and Berezin integration, respectively. The unusual form of the Laplacian in (2.7) is due to this generalization.

\section{Quantum commutators}

In the following we specialize our considerations to the case of a symplectic vector space with a Kähler structure describing a linear field theory, where $\kappa_{k l}$ is constant, cf. (2.5). For consistency this (infinite-dimensional) matrix has to be anti-Hermitian on the bosonic and Hermitian on the fermionic part of $M$. Then one can prove the following property of the projector $\Pi^{\text {hol }}$, as given by (2.4):

\section{Proposition}

Let $M$ be a linear Kähler manifold with coordinates $\left\{z_{k}\right\}_{k \in K}$ and $\mathcal{K}\left(z, z^{+}\right)=\sum z_{k}^{+} \kappa_{k l} z_{l}$ the Kähler potential, with $\kappa_{k l}$ constant. Then for any pair of polynomial observables $F$ and $G$ it holds

$$
\Pi^{\text {hol }}\left[F \Pi^{\text {hol }}[G \mid \mathcal{S}>]\right]=\Pi^{\text {hol }}\left[F \exp \left(i \hbar \overleftarrow{\partial} \cdot \vec{\partial}^{+}\right) G \mid \mathcal{S}>\right]
$$

The graded differential operator $\overleftarrow{\partial \cdot \vec{\partial}^{+}}$is given in terms of the Hermitian adjoint $\left(\kappa_{k l}^{-1}\right)^{\dagger}$ :

$$
\overleftarrow{\partial} \cdot \vec{\partial}^{+}:=\sum_{k \in K} \overleftarrow{\partial_{z_{k}}}\left(\kappa_{k l}^{-1}\right)^{\dagger} \overrightarrow{\partial_{z_{l}^{+}}}
$$

Proof :

With the Kähler potential above the Bergman kernel (2.6) becomes (cf. [Tuy1,Sch])

$$
\mathcal{B}\left(z, z^{+} ; y, y^{+}\right)=\mathcal{N}_{0} \exp \left(\frac{i}{2 \hbar} \sum_{k, l \in K} z_{k} \kappa_{k l} z_{l}^{+}-2 z_{k} \kappa_{k l} y_{l}^{+}+y_{k} \kappa_{k l} y_{l}^{+}\right)
$$

Writing $\mathcal{W}\left(z, z^{+} ; y, y^{+} ; x, x^{+}\right):=\exp \left(\frac{i \mathcal{K}\left(x, x^{+}\right)}{2 \hbar}\right) \mathcal{B}\left(z, z^{+} ; y, y^{+}\right) \mathcal{B}\left(y, y^{+} ; x, x^{+}\right)$and $\left(\frac{1}{\kappa} \partial_{y}\right)_{k}:=\sum_{l \in K} \kappa_{k l}^{-1} \partial_{y_{l}}$ this yields

$$
\begin{aligned}
& \mathcal{W}\left(z, z^{+} ; y, y^{+} ; x, x^{+}\right) y_{k}=\mathcal{W}\left(z, z^{+} ; y, y^{+} ; x, x^{+}\right)\left(i \hbar\left(\frac{1}{\kappa} \overleftarrow{\partial}_{x+}\right)_{k}+x_{k}\right) \\
& \mathcal{W}\left(z, z^{+} ; y, y^{+} ; x, x^{+}\right) y_{k}^{+}=\left(i \hbar\left(\frac{1}{\kappa} \vec{\partial}_{y}\right)_{k}+x_{k}^{+}\right) \mathcal{W}\left(z, z^{+} ; y, y^{+} ; x, x^{+}\right)
\end{aligned}
$$


Then we obtain for any monomial of the form $F_{M}\left(y, y^{+}\right)=\prod_{j \in K}\left(y_{j}^{+}\right)^{\alpha_{j}}\left(y_{j}\right)^{\beta_{j}}$ :

$$
\begin{aligned}
& \Pi^{\text {hol }}\left[F_{M} \Pi^{\text {hol }}[G \mid \mathcal{S}>]\right] \\
& =\int \mathcal{B}\left(z, z^{+} ; y, y^{+}\right)\left(\prod_{j \in K}\left(y_{j}^{+}\right)^{\alpha_{j}}\left(y_{j}\right)^{\beta_{j}} \int \mathcal{B}\left(y, y^{+} ; x, x^{+}\right) G\left(x, x^{+}\right) \mathcal{S}\left(x, x^{+}\right) d \mu_{\left(x, x^{+}\right)}\right) d \mu_{\left(y, y^{+}\right)} \\
& =\int \mathcal{W}\left(z, z^{+} ; y, y^{+} ; x, x^{+}\right) \prod_{j \in K}\left(x_{j}^{+}\right)^{\alpha_{j}}\left(i \hbar\left(\frac{1}{\kappa^{\dagger}} \vec{\partial}_{x+}\right)_{j}+x_{j}\right)^{\beta_{j}} G\left(x, x^{+}\right) \sigma(x) d \mu\left(z, x^{+}\right) d \mu_{\left(y, y^{+}\right)} \\
& =\int \mathcal{W}\left(z, z^{+} ; y, y^{+} ; x, x^{+}\right)\left(F_{M}\left(x, x^{+}\right) \sum_{n=0}^{\infty} \frac{(i \hbar)^{n}}{n !}\left(\overleftarrow{(}^{\partial} \cdot \vec{\partial}^{+}\right)^{n} G\left(x, x^{+}\right)\right) \sigma(x) d \mu\left(x, x^{+}\right) d \mu(y, y+)
\end{aligned}
$$

Here the first equality is just the definition of $\Pi^{\text {hol }}$, for the second we applied the identities for $\mathcal{W}$ and graded integration by parts [Ber] and finally we used the expansion

$$
\prod_{j \in K}\left(x_{j}\right)^{\beta_{j}} \sum_{n=0}^{\infty} \frac{1}{n !}\left(\sum_{k, l \in K} \overleftarrow{\partial}_{x_{k}}\left(\kappa_{k l}^{-1}\right)^{\dagger} \vec{\partial}_{x_{l}^{+}}\right)^{n}=\prod_{j \in K}\left(\sum_{l \in K}\left(\kappa_{j l}^{-1}\right)^{\dagger} \vec{\partial}_{x_{l}^{+}}+x_{j}\right)^{\beta_{j}}
$$

Observing that integration with $\mathcal{B}\left(y, y^{+} ; x, x^{+}\right)$is a projection, proves (3.1). . q.e.d.

The same result has also been obtained for $2 n$-dimensional Tori [BHSS]. Denoting by $\{F, G\}$ the quantum operator of the Poisson bracket between two classical observables (3.1) yields with $(2.7)$ :

\section{Theorem}

Let $M$ be a linear Kähler manifold, i.e. $\kappa_{k l}$ is a constant matrix, and $F, G$ polynomial observables, then following holds :

a) The commutator of the quantum operators $I F$ and $G$ admits an integral representation

$$
\begin{aligned}
{[\mathbb{F}, G] \| \mathcal{S}>} & =\int \mathcal{B}\left(z, z^{+} ; x, x^{+}\right) \mathcal{Q}_{[F, G]}\left(x, x^{+}\right) \mathcal{S}\left(x, x^{+}\right) d \mu_{\left(x, x^{+}\right)} \text {with } \\
\mathcal{Q}_{[F, \mathbb{E}]}\left(x, x^{+}\right) & =\left(1+\frac{i \hbar}{2} \Delta\right) F\left(x, x^{+}\right)\left(e^{i \hbar} \overleftarrow{\partial} \cdot \vec{\partial}^{+}\right)\left(1+\frac{i \hbar}{2} \Delta\right) G\left(x, x^{+}\right) \mp(\widehat{\mathrm{F} \mathrm{G}})
\end{aligned}
$$


b) The anomalous commutator is given by an expansion in $\hbar$ as

$$
\begin{aligned}
& \mathcal{A}_{[\boldsymbol{F}, \boldsymbol{G}]}\left|\mathcal{S}>:=\left(\frac{1}{i \hbar}[\boldsymbol{F}, \boldsymbol{G}]-\{F, G\}\right)\right| \mathcal{S}> \\
& =-\frac{i \hbar}{2} \sum_{k, l \in K} \int \mathcal{B}\left[\sum_{i, j \in K} \kappa_{k l}^{-1}\left(\vec{\partial}_{l}^{+} F \overleftarrow{\partial}_{i}\right) \kappa_{i j}^{-1}\left(\vec{\partial}_{j}^{+} G \overleftarrow{\partial}_{k}\right)\right] \mathcal{S} d \mu \mp(\widehat{\mathrm{F} \mathrm{G}}) \\
& -\hbar^{2}\left(\sum_{n \geq 0} \frac{(i \hbar)^{n}}{(n+3) !} \int \mathcal{B}\left[F\left(\overleftarrow{\partial}_{\vec{\partial}} \vec{\partial}^{+}\right)^{n+3} G\right] \mathcal{S} d \mu \mp(\widehat{\mathrm{F} \mathrm{G}})\right.
\end{aligned}
$$

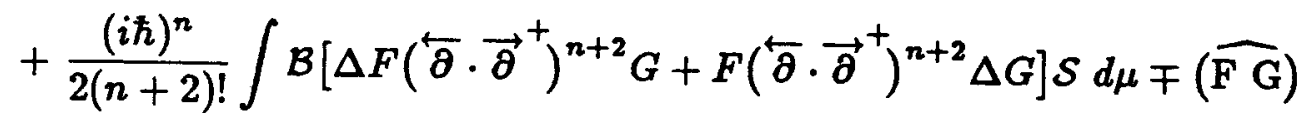

$$
\begin{aligned}
& \left.+\frac{(i \hbar)^{n}}{4(n+1) !} \int \mathcal{B}\left[\Delta F\left(\overleftarrow{\partial}^{\prime} \cdot \vec{\partial}^{+}\right)^{n+1} \Delta G\right] \mathcal{S} d \mu \mp(\widehat{\mathrm{F} \mathrm{G}})\right)
\end{aligned}
$$

Here $\mp(\widehat{F G})$ means to subtract or add the same expressions with $F, G$ interchanged for bosonic or fermionic observables, respectively. For infinite dimensions it is crucial not to interchange the order of the summations in the $\mathcal{O}(\hbar)$ term.

The first part (3.3) of the theorem indicates a possible connection between the commutator in geometric quantization and the index of the Laplace operator (2.7) - at least in the bosonic case, cf. [Gil]. It also shows up some interesting relations towards a modifcation of the geometric quantization scheme, proposed by [Tuy2]. The second part is of a more practical interest :

Obviously the construction (3.4) does not yield a representation of the full Poisson algebra, what is in accordance with the Groenewold-van Hove theorem [Gro,Jos]. Bilinear observables on finite dimensional spaces, however, should be represented correctly on quantum level. In that case the only contribution to (3.4) are the terms of order $\hbar$, but those sum up as the trace of a commutator of finite matrices and hence cancel. Here it is crucial to use the metaplectic construction, determining the coefficient of the $\operatorname{tr}^{J}$-term in (2.4) to be $-1 / 2$. Any other factor would yield only a projective representation [Woo]. Considering infinite dimensional systems the trace of a commutator need not vanish, so even the metaplectic representation of bilinears may be projective what means the appearance of field theoretic anomalies in this framework. 


\section{Constraint algebras and Schwinger terms}

Let us consider a Lie algebra $g$, acting as symmetry algebra on the phase space $(M, \omega)$ of a field theory. Then the symplectic version of Noether's theorem $[\mathrm{Ab}, \mathrm{Ma}]$ yields a set $\mathcal{C}$ of functions on $M$. Under the Poisson bracket $\mathcal{C}$ forms a Lie algebra, homomorphic to $g$, called the constraint algebra of the system. The classical dynamics is restricted to the constraint surface in $M$ and according to Dirac for all $F \in \mathcal{C}$ the corresponding quantum operators $\mathbb{I F}$ have to vanish on the Hilbert space of physical states. Thus the question arises, whether the constraint algebra closes on quantum level. Anomalous commutators between quantized constraints are called Schwinger terms and they can be computed from (3.4) :

\section{Proposition}

Let the phase space of a field theory be a symplectic vector space with a Kähler structure $\mathcal{K}\left(z, z^{+}\right)=\sum z_{k}^{+} \kappa_{k l} z_{l}$ and let a Lie algebra $g$ act on it by linear symplectic (bosonic) transformations. Then the constraint algebra $\mathcal{C}$ is spanned by quadratic polynomials

$$
F_{\mathrm{a}}=\sum_{k, l \in K}\left(z_{k} \mathbf{f}_{\mathrm{a}}{ }^{k l} z_{l}+z_{k} \mathbf{F}_{\mathrm{a}}{ }^{k l} z_{l}^{+}+z_{k}^{+} \overline{\mathbf{f}}_{\mathbf{a}}^{k l} z_{l}^{+}\right)
$$

and the Schwinger terms of the system are given as

$$
\mathcal{A}_{\left[F_{b}, F_{\mathrm{a}}\right]}=\frac{i \hbar}{2} \operatorname{tr}\left[\kappa^{-1} \mathbf{F}_{\mathbf{b}}, \kappa^{-1} \mathbf{F}_{\mathbf{a}}\right]
$$

A drawback of (4.2) lies in the potential divergence of the trace that requires a regularization. This complication may be prevented by a slightly different approach, starting with a (cohomologically trivial) central extension of the constraint algebra $\mathcal{C}$ on the classical level : Dropping the equivariance condition on the momentum map we can add to each $F_{\mathrm{a}} \in \mathcal{C}$ a constant depending linearly on $g$. Often this constants can be chosen in such a way that no divergent terms appear in the anomalous commutator (3.4) of the modified constraints. All these computations yield the same Schwinger terms, considered as element in the Lie algebra cohomology, i.e. they differ only by a functional of the commutator in $g$ [Jac]. However, only the equivariant momentum map (4.1) gives a representation of $g$ for finite dimensional systems.

An example of special interest is the non-equivariant momentum map, obtained from (4.1) by adding a term $\operatorname{tr}\left(\mathbf{F}_{\mathrm{a}}{ }^{k l}\right)$ to each constraint $F_{\mathrm{a}}$, leading to 


$$
\overline{\mathcal{A}}_{\left[F_{b}, F_{a}\right]}=\frac{i \hbar}{2} \operatorname{tr}\left(\left(2 \kappa^{-1} f_{b}\right) \cdot\left(2 \kappa^{-1} \bar{f}_{a}\right)-\left(2 \kappa^{-1} f_{a}\right) \cdot\left(2 \kappa^{-1} \bar{f}_{b}\right)\right)
$$

what often turns out to be a finite sum. Furthermore (4.3) allows an interpretation of the anomalous commutator in terms of a violation of the holomorphic structure by the Hamiltonian vector field $X_{F}$ due to the $\bar{f}$-term, cf. [BoRa]. Especially it shows that $\mathcal{A}_{\left[F_{b}, F_{b}\right]}$ is cohomologically trivial, if $\left[X_{F}, P^{J}\right] \subset P^{J}$.

(4.2) and (4.3) allow to calculate the Schwinger terms for a given field theory explicitly.

This will be demonstrated for the examples of the Kac-Moody and the Virasoro algebras in section 5 and 6 , respectively.

Another approach to eliminate unphysical degrees of freedom in a field theory with symmetries is the BRS formalism [BRS,HenTei,KoSt]. Here ghost fields are introduced, which may be considered as (classical) degrees of freedom, obeying a statistic opposite to the one of the constraints. The consistency of the BRS method requires the quantum commutator of the (trilinear) BRS-charge - containing the constraints and the ghosts with itself to vanish. Again (3.4) provides a nice tool to compute this commutator, as worked out in section 7 .

\section{Example : The Kac-Moody algebra}

To apply our methods to a physical example, we regard a system of $N$ Majorana-Weyl spinors $\Psi^{i}(\sigma)$ on the circle $S_{1}$ with the Poisson relations $\left\{\Psi^{i}(\sigma), \Psi^{j}\left(\sigma^{\prime}\right)\right\}_{+}=\delta\left(\sigma-\sigma^{\prime}\right) \delta^{i j}$. They can e.g. be thought as the independently left or right moving light cone components of $N$ noninteracting fermion fields on spacetime $S_{1} \times \mathbb{R}$, each described by a Lagrangian $\mathcal{L}=\frac{i}{2} \Psi^{T} \gamma^{0} \gamma^{\mu} \partial_{\mu} \Psi$, where $\Psi$ are real 2-component spinors [GodOli]. On these let act a gauge group $G \subset O(N)$ with local algebra $g$ represented by real antisymmetric $N \times N$ matrices $\rho^{a}$ with $\left[\rho^{a}, \rho^{b}\right]=f_{c}^{a b} \rho^{c}$, giving rise to Noether currents $J^{a}=\frac{1}{2} \sum_{i j} \rho_{i j}^{a} \Psi^{i} \Psi^{j}$

We thus get as phase space the space of sections in a $N$-dimensional real spinor bundle over $S_{1}$. Because of the required periodicity of the current observables $J^{a}(\sigma)$, the fields have to be either $2 \pi$-periodic (Ramond case) or $2 \pi$-antiperiodic (Neveu-Schwarz case) in $\sigma$. So they can be represented as

$$
\Psi^{j}(\sigma)=\sum_{r \in K} \xi_{r}^{j} e^{-i r \sigma} \quad \text { with } \begin{cases}K=\mathbb{Z}+\frac{1}{2} & \text { Neveu-Schwarz case } \\ K=\mathbb{Z} & \text { Ramond case }\end{cases}
$$

what defines anticommuting coordinates $\xi_{r}^{i}$ on the phase space, obeying $\xi_{r}^{i+}=\xi_{-r}^{i}$. 
The symplectic form corresponding to the above Poisson relations now reads

$$
\omega=\frac{1}{2} \sum_{i=1}^{N} \sum_{r \in K} d \xi_{r}^{i} d \xi_{-r}^{i}
$$

and is nondegenerated, even in the Ramond case, due to the fermionic character of the coordinates $\xi_{r}^{i}$.

The expansion of the currents $J^{a}(\sigma)$ then defines the Kac-Moody observables $T_{n}^{a}$ by

$$
J^{a}(\sigma)=\sum_{n \in \mathbb{Z}} T_{n}^{a} e^{-i n \sigma} \quad \text { and } \quad T_{n}^{a}=\sum_{i, j=1}^{N} \sum_{r, s \in K} \rho_{i j}^{a} \xi_{r}^{i} \xi_{s}^{j} \delta_{n}^{r+s}
$$

yielding the classical algebra $\left\{T_{n}^{a}, T_{m}^{b}\right\}=f_{c}^{a b} T_{n+m}^{e}$.

In the Neveu-Schwarz case we define holomorphic coordinates $z_{k}^{i}$ for $k \in \mathbb{N}$ by $z_{k}^{i}=\xi_{k-\frac{1}{2}}^{i}$ and, keeping the complex structure of the coordinates $\xi, z_{k}^{i+}=\xi_{-k+\frac{1}{2}}^{i}$. Together with the symplectic form $\omega(5.2)$ we then have a Kähler manifold with potential $\mathcal{K}\left(z, z^{+}\right)=\sum_{i} \sum_{k \in N} z_{k}^{i} z_{k}^{i+}$. The Kac-Moody observables become

$$
T_{n}^{a}=\sum_{i, j=1}^{N} \sum_{k, l \in N} \frac{\rho_{i j}^{a} \delta_{n+1}^{k+l}}{2} z_{k}^{i} z_{l}^{j}+\rho_{i j}^{a} \delta_{n}^{k-l} z_{k}^{i} z_{l}^{j+}+\frac{\rho_{i j}^{a} \delta_{-n-1}^{k+l}}{2} z_{k}^{i+} z_{l}^{j+}
$$

and we can apply (4.2) to calculate the anomalous commutator.

In the case of Ramond fields we have the additional problem to quantize the zero modes $\xi_{0}^{i}$. For the calculation of the anomaly, however, it is sufficient to require the following operator relations, inherited from the basic anticommutators :

$$
\mathbb{X}_{\xi_{n}^{i}} X_{\xi_{0}^{j}}= \begin{cases}\frac{1}{2} & \text { if } n=0 \text { and } i=j \\ \mathbb{X}_{\xi_{n}^{i} \xi_{0}^{j}} & \text { else }\end{cases}
$$

with $\mathbb{X}_{\xi}$ denoting the quantum operator corresponding to $\xi$. Using this and the derivation property of commutators and Poisson brackets the zero mode contributions to the anomaly can be shown to vanish. Then the expansion of the Kac-Moody observables $T_{n}^{a}$ then coincides up to zero mode terms with the result (5.4) for the Neveu-Schwarz case and quantizing the non-zero modes in the same way as above we are led to identical results in both cases.

Hence in either case the central term of the Kac-Moody algebra is given by (4.2)

$$
\mathcal{A}_{\left[T_{m}^{a}, T_{n}^{b}\right]}=2 i \hbar \operatorname{tr}\left[\mathbf{T}_{m}^{a}, \mathbf{T}_{n}^{b}\right] \quad \text { with } \quad\left(\mathbf{T}_{m}^{a}\right)_{i j}^{k l}=\rho_{i j}^{a} \delta_{m}^{k-l}
$$


as red off from (5.4). Thus we obtain (with $\theta_{n}$ the step function) :

$$
\begin{aligned}
\mathcal{A}_{\left[T_{m}^{a}, T_{n}^{b}\right]} & =\frac{i \hbar}{2} \sum_{i, j=1}^{N} \rho_{i j}^{a} \rho_{j i}^{b} \sum_{k \in N} \sum_{l \in N}\left(\delta_{m}^{k-l} \delta_{n}^{l-k}-\delta_{n}^{k-l} \delta_{m}^{l-k}\right) \\
& =\frac{i \hbar}{2} \sum_{i, j=1}^{N} \rho_{i j}^{a} \rho_{j i}^{b} \sum_{k}\left(\theta_{k-m}-\theta_{k-n}\right) \delta_{m}^{-n} \\
& =-\frac{i \hbar}{2} m \operatorname{tr}\left(\rho^{a} \rho^{b}\right) \delta_{m}^{-n}
\end{aligned}
$$

This coincides with the usual result for the Kac-Moody anomaly, derived e.g. with normal ordering methods [GodOli].

\section{Example : The Virasoro-Algebra}

Applying our general considerations to the example of the Virasoro algebra, we start with the bosonic loop space of all smooth embeddings of the circle $S^{1}$ into $\mathbb{R}^{(1, D-1)}$. More precisely, we consider the symplectic manifold given by

$$
\begin{aligned}
& \Omega \mathbb{R}^{(1, D-1)}:=\left\{Y^{\mu}(\sigma) \mid Y: S^{1} \rightarrow \mathbb{R}^{(1, D-1)}, Y^{\mu}(0)=0\right\} \\
& \omega=\frac{1}{2 \pi} \sum_{\mu \nu} \int_{0}^{2 \pi} d\left[Y^{\mu}(\sigma)\right] \wedge d\left[\partial_{s} Y^{\nu}(\sigma)\right] \eta_{\mu \nu} d s
\end{aligned}
$$

It can be shown, that the phase space $\left(\Omega \mathbb{R}^{(1, D-1)}, \omega\right)$ is isomorphic to the canonical symplectic manifold $T^{*} Q$ [BoRa], where $Q$ denotes the space of embeddings of the intervall $[0, \pi]$ into $\mathbb{R}^{(1, D-1)} \cdot T^{*} Q$ is the classical phase space of the open bosonic string. The Virasoro algebra arises as the quantization of the Lie algebra gDiff of infinitesimal reparametrizations of $S^{1}$ : The generators of the corresponding constraint algebra $\mathcal{C}_{\text {Diff }}$ are

$$
L(\sigma)=\sum_{\mu \nu=1}^{D} \partial_{\sigma} Y^{\mu}(\sigma) \partial_{\sigma} Y^{\nu}(\sigma) \eta_{\mu \nu}
$$

Fourier expansion of the fields $Y^{\mu}(\sigma)$ and the constraints (6.2) yields

$$
\begin{aligned}
\omega & =\sum_{k, l \in N} \sum_{\mu, \nu=1}^{D} 2 i k \delta_{k}^{l} \eta_{\mu \nu} d z_{k}^{\mu} d z_{l}^{\nu+} \\
L_{N} & =\sum_{k \neq 0} \sum_{\mu, \nu=1}^{D} Y_{k}^{\mu} i k(k+N) \eta_{\mu \nu} Y_{-N-k}^{\nu}
\end{aligned}
$$


These $L_{N}$, the Virasoro generators of $\mathcal{C}_{\mathrm{Diff}}$, constitute the classical Poisson algebra $\left\{L_{M}, L_{N}\right\}=(M-N) L_{M+N}$ that is quantized geometrically using (2.7). The Fourier modes $Y_{k}^{\mu}$ of the fields $Y^{\mu}(\sigma)$ induce in a natural way a Kähler structure on $\left(\Omega \mathbb{R}^{(1, D-1)}, \omega\right)$ with

$$
z_{k}^{\mu}=Y_{k}^{\mu} \quad \text { and } \quad z_{k}^{\mu+}=Y_{-k}^{\mu} \quad \text { for } \quad k \in \mathbb{N}
$$

as holomorphic coordinates. Then the Virasoro generators can be written as

$$
L_{N}=\sum_{k, l \in N} z_{k}^{\mu}\left(\lambda_{N}\right)_{\mu \nu}^{k l} z_{l}^{\nu}+z_{l}^{\nu}\left(\mathbf{L}_{\mathrm{N}}\right)_{\mu \nu}^{k l} z_{l}^{\mu+}+z_{k}^{\mu+}\left(\bar{\lambda}_{N}\right)_{\mu \nu}^{k l} z_{l}^{\mu+}
$$

and we can work out the anomaly of the constraint algebra from the formulas of section 2 : The coefficient matrix $\mathbf{L}_{\mathbf{N}}$ is

$$
\left(\mathbf{L}_{\mathbf{N}}\right)_{\mu \nu}^{k l}= \begin{cases}2 i k(k+N) \delta_{k+N}^{l} \eta_{\mu \nu} & N \geq 0 \\ 2 i l(l-N) \delta_{l-N}^{k} \eta_{\mu \nu} & N \leq 0\end{cases}
$$

with $k, l>0$. Clearly the anomalous commutator vanishes for $N \cdot M \geq 0$, thus we demand without loss of generality $N>0$ and $M<0$. From (4.2) we obtain

$$
\begin{aligned}
\mathcal{A}_{\left[\boldsymbol{L}_{M}, \boldsymbol{L}_{N}\right]} & =\frac{i \hbar}{2} \operatorname{tr}\left(\sum_{n \in \boldsymbol{N}} n(n+N) \delta_{n-M}^{k} \delta_{n+N}^{l}-(\widehat{\mathrm{M} \mathrm{N}})\right) \\
& =D \frac{i \hbar}{2} \sum_{k \in \boldsymbol{N}}\left(k(k-N) \theta_{k-N}-k(k-M) \theta_{k}\right) \delta_{M}^{-N}
\end{aligned}
$$

This yields the Virasoro-Algebra

$$
\left[\mathbb{L}_{M}, \mathbb{L}_{N}\right]=i \hbar(M-N) \mathbb{L}_{M+N}-\frac{D}{12} \hbar^{2}\left(N^{3}+(12 \beta-1) N\right) \delta_{M}^{-N}
$$

as the central extended constraint algebra $\mathcal{C}_{\text {Diff }}$. The (divergent) constant $\beta=\sum_{k \in N} k$ may be removed by a redefinition of the generator $L_{0}$. We note that the central term, linear in $N$, is cohomologically trivial. The anomaly may also be computed in a cohomological equivalent form from (4.3): Determining the respective coefficient matrices $\lambda_{N}$ and $\bar{\lambda}_{N}$ from (6.3) we obtain

$$
\begin{aligned}
\tilde{\mathcal{A}}_{\left[\boldsymbol{L}_{M}, \boldsymbol{L}_{N}\right]} & =2 i \hbar \operatorname{tr}\left(\left(\kappa^{-1} \lambda_{M}\right) \cdot\left(\kappa^{-1} \bar{\lambda}_{N}\right)\right) \\
& =D \frac{i \hbar}{2}\left(\sum_{k \in N}(N-k)(N+M+k) \theta_{N-k}\right) \delta_{M}^{-N} \\
& =\frac{D}{12} i \hbar\left(N^{3}-N\right) \delta_{M}^{-N}
\end{aligned}
$$


In the latter construction the anomaly appears without divergences, but the constraint algebra $\mathcal{C}_{\text {Diff }}$ acquires an extension already on the classical level. $A$ similar result has been derived by [BoRa], who, however, omitted the half form contributions, leading to a failure of the representation already for finite dimensional systems.

\section{The BRS-Operator and Geometric Quantization}

In the context of geometric quantization the BRS-scheme [BRS] may be described as follows [KoSt,HenTei]: The phase space $(M, \omega)$ of a classical system with an algebra $\mathcal{C}$ of first class constraints (identified in the following with the symmetry algebra $g$ ) is extended by introducing the generators of the exterior algebras $\wedge \sigma$ and $\wedge g^{*}$ as additional fermionic coordinates (ghost variables). For a basis $\left\{g_{i}\left(z, z^{+}\right)\right\}_{i \in I}$ of $g$ we denote the structure constants by $f_{i j}^{k}$ and the generators of $\wedge g^{*}$ and $\wedge g$ by $c^{i}$ (dual to $g_{i}$ ) and $\pi_{i}$ (dual to $c^{i}$ ), respectively. Then

$$
\omega_{\mathrm{ext}}=\omega+\omega_{g h o s t}=\omega+\sum_{j \in I} d \pi_{j} d c^{j}
$$

is a symplectic form on the extended phase space $M_{\text {ext }}$.

[In the notion of [Kos] $\wedge g^{*}$ is a sheaf over a pointlike manifold and thus defines a graded manifold, which may be seen as the configuration space of the ghost sector. In this setting $\wedge g \otimes \wedge g^{*}$ plays the role of the cotangent bundle over $\wedge g^{*}$ and $\omega_{g h o s t}$ is the corresponding canonical two form. Then $M_{\text {ext }}$ is to be written as the graded manifold $\left.\left(M, C^{\infty}(M) \otimes \wedge g^{*} \otimes \wedge g\right).\right]$

The symplectic action of the ghost number charge $N_{g h o s t}=\sum \pi_{j} c^{j}$ (defined by the Poisson bracket $\left\{N_{\text {ghost }}, \cdot\right\}$ ) induces a $\mathbb{Z}$-grading on the space of functions over $M_{\text {ext }}$. The BRS-charge

$$
Q\left(z, z^{+}, c, \pi\right)=\sum_{j \in K} c^{j} g_{j}\left(z, z^{+}\right)-\frac{1}{2} \sum_{i, j, k \in K} c^{i} c^{j} f_{i j}^{k} \pi_{k}
$$

has ghost number -1 and its symplectic action (defining the classical BRS operator) is nilpotent, i.e. $\{Q,\{Q, \cdot\}\}=0$. Thus this operator defines a cohomology. It can be shown [KoSt,DuElTu,HenTei] that the zero'th cohomology class of the classical BRS operator is isomorphic (with respect to the Poisson algebras) to the space of functions on the reduced phase space (i.e. the constraint surface modulo the action of the symmetry group generated by the constraints). Thus as physical sector of the quantum Hilbert space one naturally 
takes the zero'th cohomology class of the quantized BRS operator $\varphi$. This, obviously, requires $Q^{2}=0$.

Therefore, starting from a bilinear constraint algebra and assuming a Kähler structure on $M_{\text {ext }}$, we consider the fermionic trilinear BRS charge (7.2), obeying $\{Q, Q\}=0$, and calculate $Q^{2}$ either from (3.4)

$$
\begin{aligned}
\frac{1}{i \hbar} Q^{2}|\mathcal{S}\rangle= & \frac{1}{2} \mathcal{A}_{[Q, Q]} \mid \mathcal{S}> \\
= & -\frac{i \hbar}{2} \sum_{k, l \in K} \int \mathcal{B}\left[\sum_{i, j \in K} \kappa_{k l}^{-1}\left(\vec{\partial}_{l}^{+} Q \overleftarrow{\partial}_{i}\right) \kappa_{i j}^{-1}\left(\vec{\partial}_{j}^{+} Q \overleftarrow{\partial}_{k}\right)\right] \mathcal{S} d \mu \\
& -\hbar^{2} \int \mathcal{B}\left[Q \frac{\left(\overleftarrow{\partial} \cdot \vec{\partial}^{+}\right)^{3}}{6} Q+\Delta Q \frac{\overleftarrow{\partial}_{\vec{\partial}} \cdot \vec{\partial}^{+}}{4} \Delta Q\right] \mathcal{S} d \mu
\end{aligned}
$$

or directly from (3.3) as

$$
\frac{1}{i \hbar} Q^{2} \mid \mathcal{S}>=\frac{i \hbar}{2} \int \mathcal{B}\left[\Delta Q \overleftarrow{\partial} \cdot \vec{\partial}^{+} Q+Q \overleftarrow{\partial} \cdot \vec{\partial}^{+} \Delta Q+Q\left(\overleftarrow{\partial} \cdot \vec{\partial}^{+}\right)^{2} Q\right] \mathcal{S} d \mu+\mathcal{Q}\left(\hbar^{2}\right)
$$

Choosing a suitable base on $\sigma^{\mathscr{C}}$, the corresponding set of (complex) ghost coordinates $\left\{c^{i}, \pi_{i}\right\}$ splits completely into a holomorphic and an antiholomorphic part. Then, as worked out in the appendix, graphical methods allow to prove:

i) The terms of order $\hbar^{2}$ in (7.3) vanish.

ii) $(7.3 \mathrm{a})$ reduces to

$$
\frac{1}{i \hbar} Q^{2} \mid \mathcal{S}>=\frac{1}{2} \int \mathcal{B}\left[\sum_{i j \in I} c^{i} c^{j} \mathcal{A}_{\left[g_{i}, g_{j}\right]}+2\left(\sum_{k} \sum_{l}\right)^{\prime} f_{i k}^{l} f_{j l}^{k}\right] \mathcal{S} d \mu
$$

$\mathcal{A}_{\left[g_{i}, g_{j}\right]}$ is given by (3.4) and the symbol $\left(\sum_{k} \sum_{l}\right)^{\prime}$ means that the summation is restricted to those $k$ and $l$, for which $c^{k}$ and $c^{l}$ are both holomorphic or both antiholomorphic (We note that the order of the summations is still important). Of course, using (7.1) and (7.3a) an algebraic proof of (7.4) is also possible.

Specializing now to the case of the Virasoro algebra as the constraint algebra of the bosonic string, (7.1) and (7.2) yield the symplectic form

$$
\omega_{e x t}=\omega+\sum_{N \in z} d \pi_{N} d c^{-N}
$$


and the (trilinear) BRS-charge

$$
Q_{V}=\sum_{N \in \mathbb{Z}} c^{-N} L_{N}\left(z, z^{+}\right)-\frac{1}{2} \sum_{M, N \in \mathbb{Z}} c^{-M} c^{-N}(M-N) \pi_{M+N}
$$

respectively, where $\omega$ and $L_{N}$ are defined as in (6.1). The $\mathrm{c}^{N}$ and $\pi_{N}$ may be regarded as Fourier components of ghost fields $\mathrm{C}(\sigma), \pi(\sigma)$ corresponding to the basis (6.2) in $\sigma$ and thus the holomorphic structure (6.4) on $\mathrm{M}$ has a natural extension on $\mathrm{M}_{\text {ext }}$ :

$$
\begin{aligned}
z_{N}^{c} & =c^{N} & z_{N}^{\pi} & =\pi_{N} \\
z_{N}^{c}+ & =c^{-N} & z_{N}^{\pi^{+}} & =\pi_{-N}
\end{aligned} \quad \text { for } \quad N \in \mathbb{N}
$$

In addition we may choose $z_{0}^{c}=c^{0}$ and $\left(z_{0}^{c}\right)^{+}=\pi_{0}^{\dagger}$

Now (7.4) yields

$$
\begin{aligned}
\frac{1}{i \hbar} Q_{V}^{2} & =\frac{1}{2} \sum_{M, N \in \mathbb{Z}} c^{\left.-M^{-N} c^{-N} \mathcal{H}_{M}, \boldsymbol{L}_{N}\right]} \\
& -i \hbar \sum_{N \in N} c^{-N} c^{N}\left(\sum_{K=0}^{\infty}(2 N+K)(K-N)-\sum_{K=N}^{\infty}(N+K)(K-2 N)\right)
\end{aligned}
$$

alternatively using $(7.3 \mathrm{~b})$ we arrive at

$$
\begin{aligned}
\frac{1}{i \hbar} Q_{V}^{2} & =\sum_{M, N \in \mathbb{Z}} c^{-M} c^{-N} \tilde{\mathcal{A}}_{\left[\boldsymbol{L}_{N}, \mathbf{L}_{N}\right]} \\
& -i \hbar \sum_{N \in \boldsymbol{N}} c^{-N^{N} c^{N}}\left(N(2-D) \sum_{K=0}^{\infty} K-\sum_{K=0}^{N-1}(N+K)(2 N-K)\right)
\end{aligned}
$$

$\mathcal{A}_{\left[\boldsymbol{L}_{M}, \boldsymbol{L}_{N}\right]}$ and $\overline{\mathcal{A}}_{\left\{\mathbb{L}_{M}, \boldsymbol{L}_{N}\right]}$ are given by (6.7) and (6.9), respectively. Calculating the infinite sum in (7.8b) with the same treatment of divergences as used for (6.8), we see both expressions (7.8) to be equivalent to

$$
\frac{1}{i \hbar} Q_{V}^{2}=-i \hbar \sum_{N \in N} c^{-N} c^{N}\left(\frac{D}{12}\left(N^{3}-N\right)-N \beta D+2 N \beta-\frac{13}{6} N^{3}+\frac{1}{6} N\right)
$$

The term proportional to $N^{3}$ vanishes for $D=26$, the critical dimension of the bosonic string, while the term proportional to $N$ may be removed by a redefinition of $L_{0}$ on the classical level.

$\dagger$ Strictly speaking the last definition will not yield a complex structure in the ghost sector, but it defines a polarisation in $T^{\mathbb{C}} M_{\text {ext }}$, as needed for geometric quantization. 


\section{Appendix}

For the evaluation of (3.4) a graphical representation reminding the technique of Feynman graphs in perturbation theory may be useful:

We represent each monomial of degree $n$ by a vertex with $n$ (oriented) external legs and the operator $\overleftarrow{\partial} \cdot \vec{\partial}^{+}$by a "propagator line" (see fig.1).

The method is best illustrated by an example:

For a bilinear constraint algebra the graph for the BRS charge $Q$ (7.2) looks like in fig.2 and we have the "propagators" indicated in fig.3. We see that $\Delta Q$ contains only terms with external $c$-line (fig.4). As no $c-c$ propagator exists, the last term in (7.3a) (fig.5) vanishes.

With similar arguments we see that the other contribution of order $\hbar^{2}$ in (7.3), as shown in fig.6, also vanishes. So only the $\mathcal{O}(\hbar)$ terms contribute in (7.3) and we have fig.7 for the rest of (7.3a) yielding (7.4).

Furthermore the identity $\{Q, Q\}=0$, used in section 7 is for any trilinear $Q$ represented by the graph in fig.8. Applying $\Delta$ to it yields fig.9, which shows the $\mathcal{O}(\hbar)$ terms of (7.3a) and $(7.3 \mathrm{~b})$ to be indeed identical. 


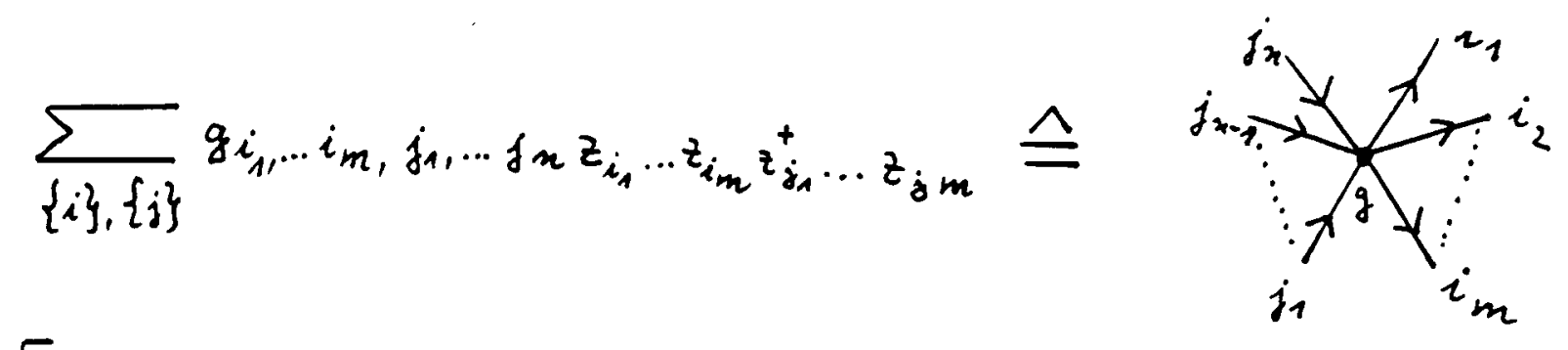

Fig. 1

$$
\partial_{i} \vec{\partial}_{j}^{+} \triangleq \stackrel{k_{i j}^{-k}}{\longrightarrow}
$$

Fig.2 $Q \hat{=}$

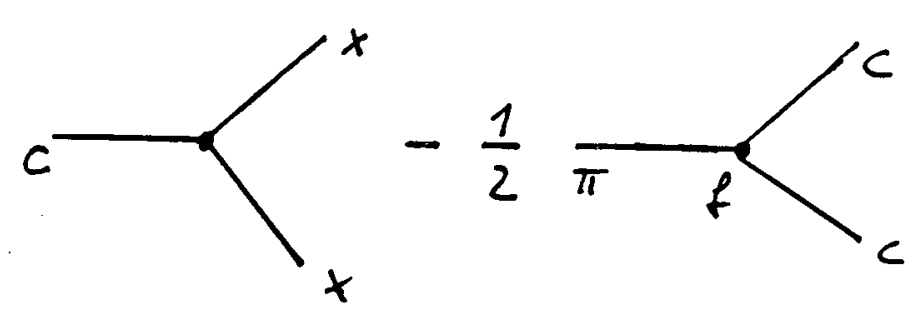

(x coordinate on M)

Fig. 3
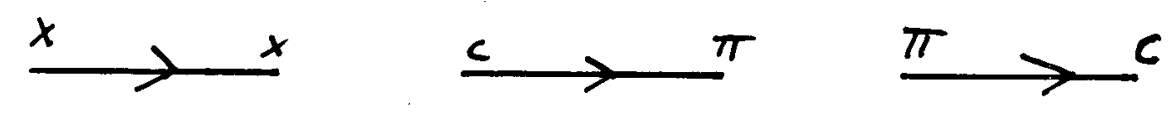

Fig.4 $\Delta Q \triangleq$

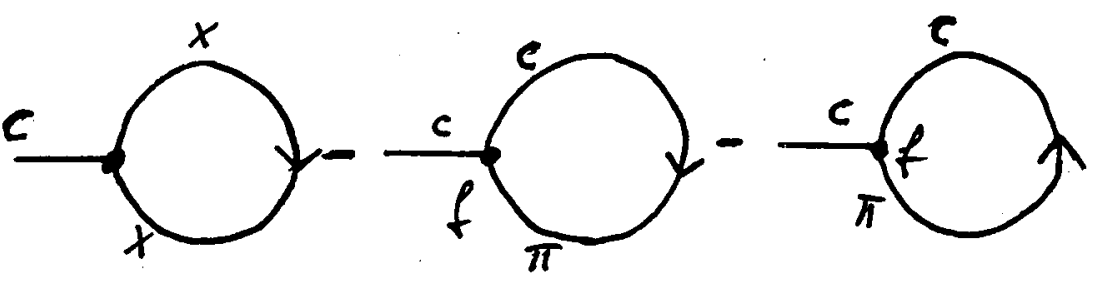

Fig. 5

$0=$

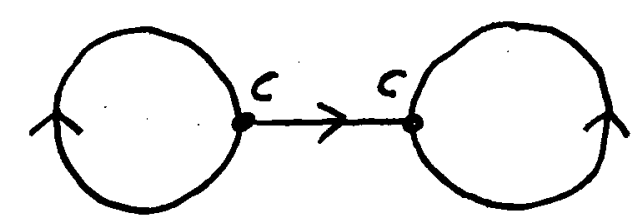


Fig.6 $0=$

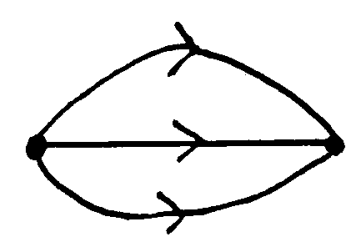

Fig.7 $Q^{2}=S_{x}^{x}+\int_{\pi}^{c} c+C_{c}^{c} c$

$\underline{\text { Fig. } 8}\{Q, Q\} \hat{=} \longrightarrow<=0$

Fig. 9
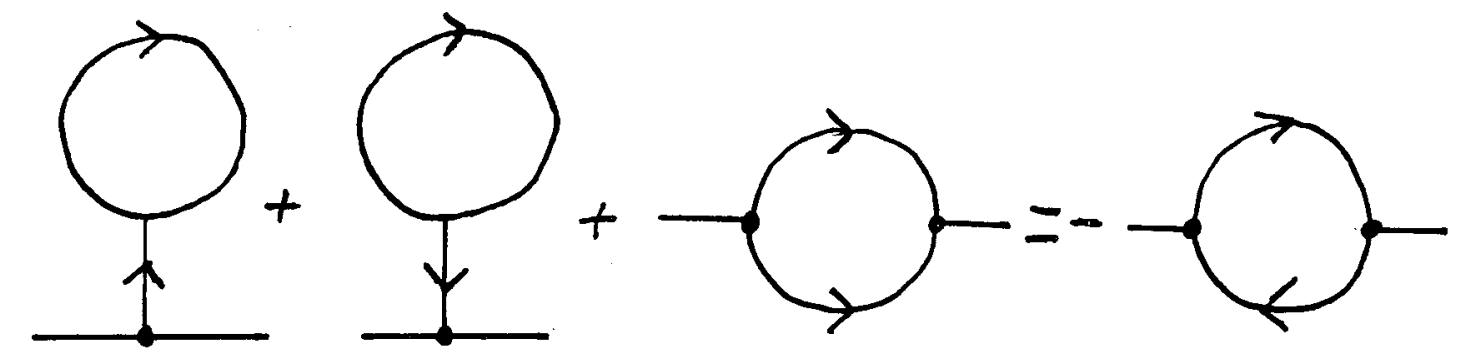

17 


\section{List of References}

[AbMa] R.Abraham and J.E.Marsden, Foundations of Mechanics (2nd Edition, Benjamin, New York, 1975).

[Bar] V.Bargmann, Comm. Pure and Appl. Math. 14 (1961) 187.

[Ber] F.A.Berezin, Introduction to Superanalysis (MPAM 9, Reidel, Dordrecht, 1987).

[BoRa] M.J.Bowick and S.G.Rajeev, Nucl. Phys. B296 (1988) 1007; Nucl. Phys. B293 (1987) 348.

[Bla] R.J.Blattner, Proc. Symp.Pure Math. 26 (1983) 147.

[BRS] C.Becchi, A.Rouet and R.Stora, Phys. Lett B52 (1974) 344; Ann. Phys, 98 (1976) 287.

[BHSS] M.Bordemann, J.Hoppe, P.Schaller and M.Schlichenmeier, Karlsruhe preprint (KA-THEP 4-90).

[DuElTu] C.Duval, J.Elhadad and G.M.Tuynman, Marseille Preprint (CPT-89/P.2248)

[Gil] P.B.Gilkey, Invariance Theory, the Heat Equation and the AthiyahSinger Index Theorem (Publish or Perish, Boston, 1984).

[GodOli] P.Goddard and D.Olive, Int. J. Mod. Phys. A1 (1986) 303.

[Gro] H.J.Groenewold, Physica 12 (1946) 405.

[GuSt] V.Guillemin and S.Sternberg, Geometric Asymptotics (American Mathematical Society, Providence, 1977).

[HenTei] M.Henneaux and C.Teitelboim, Comm. Math. Phys. 115 (1988) 213.

[Kos] B.Kostant, Graded Manifolds, Graded Lie Theory and Prequantization (in : Lecture Notes Mathematics 579, Ed : K.Bleuler and A.Reetz, Springer, Heidelberg, 1977).

[KoSt] B.Kostant and S.Sternberg, Ann. Phys. 176 (1987) 49.

[Jac] N.Jacobson, Lie Algebras (Wiley and Sons, New York, 1962).

[Jos] A.Joseph, Comm. Math. Phys. 17 (1970) 210. 
[Nie] A.J.Niemi, Ann. Phys. 187 (1988) 369.

[PIWa] K.Pilch and N.P.Warner, Class. Quantum Grav. 4 (1987) 1183.

[SaSw] P.Schaller and G.Schwarz, Karlsruhe Preprint (KA-THEP 3-89), submitted to J. Math. Phys.

[Sch] G.Schwarz, Geometrische Quantisierung von Feldtheorien und deren Anomalien, Karlsruhe thesis (1989).

[Sni] J.Śniatycki, Geometric Quantization and Quantum Mechanics (Springer, New York, 1980).

[Tuy1] G.M.Tuynman, J. Math. Phys. 28 (1987) 573.

[Tuy2] G.M.Tuynman, J. Math. Phys. 28 (1987) 2829.

[Woo] N.Woodhouse, Geometric Quantization (Clarendon, Oxford, 1980). 